\title{
OPTIMAL GENERALIZED LOGARITHMIC MEAN BOUNDS FOR THE GEOMETRIC COMBINATION OF ARITHMETIC AND HARMONIC MEANS
}

\author{
Bo-Yong LONG ${ }^{1}$ AND Yu-Ming $\mathrm{CHU}^{2}$ \\ ${ }^{1}$ College of Mathematics Science, Anhui University, Hefei 230039, P. R. \\ China, lbymxy@163.com \\ ${ }^{2}$ Department of Mathematics, Huzhou Teachers College, Huzhou 313000, P. \\ R. China, chuyuming@hutc.zj.cn
}

\begin{abstract}
In this paper, we answer the question: for $\alpha \in(0,1)$, what are the greatest value $p=p(\alpha)$ and least value $q=q(\alpha)$, such that the double inequality $L_{p}(a, b) \leq A^{\alpha}(a, b) H^{1-\alpha}(a, b) \leq L_{q}(a, b)$ holds for all $a, b>0$ ? where $L_{p}(a, b)$, $A(a, b)$, and $H(a, b)$ are the $p$-th generalized logarithmic, arithmetic, and harmonic means of $a$ and $b$, respectively.

Key words: Generalized logarithmic mean, arithmetic mean, harmonic mean.
\end{abstract}

2000 Mathematics Subject Classification: 26E60.

Received: 05-05-2011, revised:12-10-2011, accepted: 12-10-2011. 